\title{
SOME RESULTS ON ESSENTIAL SPECTRA OF DIFFERENTIAL OPERATORS IN BANACH SPACES
}

\section{V.A. EROVENKO}

Department of Mathematics and Mechanics, Belarusian State University

F. Skoriny av. 4, 220050 Minsk, Belarus

E-mail: erovenko@bsu.by

Received June 5, 2002; revised April 9, 2003

\begin{abstract}
In this paper we investigate spectral and semi-Fredholm properties of maximum and minimum Fuchsian differential operators on Lebesgue spaces on a semi-axis. These results are applied for determination of various essential spectra and spectrum of ordinary differential operators with polynomial coefficients, which order does not exceed the order of the corresponding derivative.
\end{abstract}

Key words: spectral properties, Fuchsian differential operators, Fredholm and semiFredholm operators, Lebesgue spaces

\section{INTRODUCTION}

As a rule for certain differential equations the functional spaces in which the operators, corresponding to considered equations, are not defined uniquely by conditions of a problem. Therefore the theorems devoted to spectral, Fredholm and semi-Fredholm properties of operators on Lebesgue spaces are of interest.

For the first time spectra of differential operators of the second order with polynomial coefficients, which order does not exceed the order of corresponding derivative, was studied in the paper of Rota [18]. Balslev and Gamelin [2] have investigated in detail the Fredholm properties of maximum differential operators generated by Fuchsian differential expression such as

$$
(m f)(t)=\sum_{j=0}^{n} a_{j}(t) f^{(j)}(t)
$$


where $a_{k}(t)=O\left(t^{k}\right)$ on $L^{p}(1, \infty), 1<p<\infty$.

For the closed linear operator $T$ on a complex Banach space $X$ the essential spectra of operator $T$ can be defined as a complement of the set with respect to the complex plane $\mathbf{C}$, defined by various Fredholm properties of a family of operators $T-\lambda I$ :

$$
\begin{aligned}
\sigma_{e k}(T):=C \backslash \Delta_{k}(T), & k=1,2,3,4,5, \\
\sigma_{e 2}^{+}(T):=C \backslash \Phi^{+}(T), & \sigma_{e 2}^{-}(T):=C \backslash \Phi^{-}(T),
\end{aligned}
$$

where

$$
\begin{aligned}
& \Delta_{1}(T):=\{\lambda \in C: \overline{R(T-\lambda I)}=R(T-\lambda I)\} \\
& \Phi^{+}(T):=\left\{\lambda \in \Delta_{1}(T): \operatorname{nul}(T-\lambda I)<\infty\right\} \\
& \Phi^{-}(T):=\left\{\lambda \in \Delta_{1}(T): \operatorname{def}(T-\lambda I)<\infty\right\} \\
& \Delta_{2}(T):=\Phi^{+}(T) \cup \Phi^{-}(T)=s-\Phi(T) \\
& \Delta_{3}(T):=\Phi^{+}(T) \cap \Phi^{-}(T)=\Phi(T) \\
& \Delta_{4}(T):=\left\{\lambda \in \Delta_{3}(T): \operatorname{ind}(T-\lambda I)=0\right\}=\Phi_{0}(T), \\
& \Delta_{5}(T):=\left\{\lambda \in \Delta_{4}(T): \text { a deleted neighborhood of } \lambda \text { in the resolvent set }\right\} .
\end{aligned}
$$

Each set $\sigma_{e k}(T), k=\overline{1,5}$, and $\sigma_{e 2}^{ \pm}(T)$ is called an essential spectrum. It is clear that $\sigma_{e k}(T) \subseteq \sigma_{e l}(T)$ for $k \leq l$ and $\sigma_{e 2}(T) \subseteq \sigma_{e 2}^{ \pm}(T) \subseteq \sigma_{e 3}(T)$, moreover inclusions can be eigen. In the modern mathematical literature the essential spectra are called by names of Goldberg $\sigma_{e 1}(T)$, Kato $\sigma_{e 2}(T)$, Wolf $\sigma_{e 2}^{+}(T)$, Gustafson-Weidmann $\sigma_{e 2}^{-}(T)$, Fredholm $\sigma_{e 3}(T)$, Weyl $\sigma_{e 4}(T)$ and Browder $\sigma_{e 5}(T)$. Various characteristic properties of the stability of essential spectra under relatively compact, relatively small perturbations, or polynomial mappings and also same equivalent methods of their descriptions are given in papers $[3 ; 12 ; 13 ; 17 ; 20]$.

\section{EULER DIFFERENTIAL OPERATION}

Let us consider Fuchsian differential operation which can be considered as a perturbed Euler differential operation:

$$
\omega:=\sum_{k=0}^{n}\left(a_{k}+b_{k}(t)\right) t^{k} D^{k}, \quad a \leq t<\infty, 0<a<\infty,
$$

where $b_{k}(t)$ are complex-valued functions such that $b_{k}(t) \in C^{k}[a, \infty), a_{k}$ are complex numbers, and the following conditions are satisfied

$$
a_{n} \neq 0, \quad 1 /\left(a_{n}+b_{n}\right), b_{k} \in L^{\infty}(a, \infty), \quad 0 \leq k \leq n,
$$


Some results on essential spectra of diff. operators in Banach spaces 205

here $D:=d / d t$. A differential expression $\varepsilon:=\sum_{k=0}^{n} a_{k} t^{k} D^{k}$ with corresponding degree coefficients is called the Euler operation [2]. Let us denote $T(\omega, p,[a, \infty)), 0<a<\infty$, a maximal operator, which is defined on $L^{p}(a, \infty)$ in the following way:

$$
D[T(\omega, p,[a, \infty))]:=\left\{f: f^{(n-1)} \in A C_{l o c}[a, \infty) ; f, \omega f \in L^{p}(a, \infty)\right\},
$$

where $A C_{l o c}[a, \infty)$ is a set of complex-valued functions $f$, absolutely continuous in each compact sub-interval $[a, \infty)$, and

$$
T(\omega, p,[a, \infty)) f:=\omega f \quad \text { for } \quad f \in D[T(\omega, p,[a, \infty))] .
$$

Denote $T_{0}(\omega, p,[a, \infty)), 0<a<\infty$, a minimal operator, defined on $L^{p}(a, \infty)$ for $1 \leq p<\infty$ as a closure of a restriction of maximal operator $T(\omega, p,[a, \infty))$ on a set of functions from $D[T(\omega, p,[a, \infty))]$, having the compact support in $(a, \infty)$, and for $1<p \leq \infty$ it is defined through Banach conjugate operator $T^{\prime}\left(\omega^{*}, p^{\prime},[a, \infty)\right)$, where $\omega^{*}$ is a formally adjoint differential operation:

$$
\omega^{*} g:=\sum_{k=0}^{n}(-1)^{k} D^{k}\left(\left(a_{k}+b_{k}(t)\right) t^{k} g\right), a \leq t<\infty
$$

and

$$
1 / p+1 / p^{\prime}=1 \text {, if } 1<p<\infty, \quad p^{\prime}=\infty \text {, if } p=1, \quad p^{\prime}=1 \text {, if } p=\infty
$$

Spectral, Fredholm and semi-Fredholm properties of minimal and maximal ordinary differential operators with constant, almost constant coefficients, and Euler differential operators on Lebesgue spaces are investigated in $[5 ; 6 ; 8]$.

Denote by $\gamma(T)$ the Kato minimum module for closed linear operator $T$ ([13], section IV.5.1), i.e.:

$$
\gamma(T):=\inf \left\{\frac{\|T u\|}{\operatorname{dist}(u, N(T))}: u \in D(T), u \notin N(T)\right\}
$$

where $N(T)$ is a zero-space, being a closed subspace. In the other words, $\gamma(T)$ is the greatest number $\gamma$ such that

$$
\|T u\| \geq \gamma \operatorname{dist}(u, N(T)), \quad u \in D(T) .
$$

Note, that if the closed operator $T: X \rightarrow Y$, where $X, Y$ are Banach spaces, then the range of values $R(T)$ is closed if and only if $\gamma(T)>0$ ([13], Theorem IV.5.2 and [12], Theorem IV.1.6). 


\section{OPERATOR'S SPECTRA}

The central place in further research of subsets of perturbed operator spectra is given by the theorem on the stability of semi-Fredholm operators (see, e.g., [13], Theorem IV.5.22 and [12], Theorem V.3.6).

Lemma 3.1. Let $X$ and $Y$ are Banach spaces, $T: X \rightarrow Y$ is a closed linear operator, $B: X \rightarrow Y D(T) \subset D(B)$. If $T$ is a semi-Fredholm operator, i.e. $\overline{R(T)}=R(T), \operatorname{nul}(T)<\infty$ or def $(T)<\infty$, and

$$
\|B x\| \leq a\|x\|+b\|T x\|, \quad x \in D(T),
$$

where $a$ and $b$ are non-negative numbers such that $a+b \gamma(T)<\gamma(T)$ (i.e., $B$ is $T$-bounded operator), then the operator $T+B$ is closed and semi-Fredholm and

$$
n u l(T+B) \leq \operatorname{nul}(T), \quad \operatorname{def}(T+B) \leq \operatorname{def}(T), \quad \operatorname{ind}(T+B)=\operatorname{ind}(T) .
$$

The following theorem is the base for the study of spectral properties of differential operators. The proof of this theorem is based on Lemma 3.1 and principle of decomposition for essential spectra of ordinary differential operators [5].

For its formulation let us consider a formal differential operation which is given in a general form:

$$
\mu:=\tau+\sum_{k=0}^{n} b_{k}(t) D^{k}=\sum_{k=0}^{n}\left(a_{k}(t)+b_{k}(t)\right) D^{k}, a \leq t<\infty
$$

where $\tau:=\sum_{k=0}^{n} a_{k}(t) D^{k}$ and $a_{k}(t), b_{k}(t)$ are such complex-valued functions, that $a_{k}, b_{k} \in C^{k}(a, \infty), b_{k}, 1 /\left(a_{n}+b_{n}\right) \in L^{\infty}(a, \infty), k=\overline{0, n}$. Maximal and minimal differential operators $T(\mu, p,[a, \infty)), T_{0}(\mu, p,[a, \infty))$, and $T(\tau, p,[a, \infty)), T_{0}(\tau, p,[a, \infty))$ are generated by formal differential operations $\mu$ and $\tau$, respectively, and they are defined similarly to operators for operation $\omega$, defined by formula $(2.1)$.

Theorem 3.1. Let coefficients $b_{k}(t), 0 \leq k \leq n$ in (3.1) satisfy integral conditions of a smallness on infinity

$$
\sup _{m \leq s<\infty} \int_{s}^{s+1}\left|b_{k}(t)\right|^{p} d t \rightarrow 0 \text { where } m \rightarrow \infty, \quad 0 \leq k \leq n-1 .
$$

Then for essential spectra of minimal operator $T_{0}(\mu, p,[a, \infty))$ and maximal operator $T(\mu, p,[a, \infty))$, generated by differential operation $\mu$ on Banach spaces $L^{p}(a, \infty),-\infty<a<\infty, 1 \leq p<\infty$, and for closed differential 
Some results on essential spectra of diff. operators in Banach spaces 207

operator $S(\mu, p,[a, \infty))$, being extension of minimal and restriction of maximal operators, i.e. satisfying inclusion

$$
T_{0}(\mu, p,[a, \infty)) \subset S(\mu, p,[a, \infty)) \subset T(\mu, p,[a, \infty)),
$$

the following equalities are valid:

$$
\begin{aligned}
& \sigma_{e k}[S(\mu, p,[a, \infty))]=\sigma_{e k}[S(\tau, p,[a, \infty))], k=1,2,2^{ \pm}, 3 \\
& \sigma_{e k}\left[T_{0}(\mu, p,[a, \infty))\right]=\sigma_{e k}\left[T_{0}(\tau, p,[a, \infty))\right], k=4,5 \\
& \sigma_{e k}[T(\mu, p,[a, \infty))]=\sigma[T(\tau, p,[a, \infty))], k=4,5
\end{aligned}
$$

Let us note that using additional assumptions on "smallness in average on infinity" for derivative $b_{k}^{(i)}(t), 1 \leq k \leq n, 1 \leq i \leq k$, such as (3.2) with index $p=1$, and using the preconjugate operators, we can prove correctness of the formulas for essential spectra of ordinary differential operators from Theorem 3.1 also in the space $L^{\infty}(a, \infty), 0<a<\infty$.

Under special "smallness on infinity" conditions for coefficients $b_{k}(t)$ it is possible to reduce differential operators, generated by perturbed differential Euler operation $\omega$, to ordinary differential operators with almost constant coefficients and then to apply the statement of Th. 3.1 on the invariance of essential spectra. Coincidence of essential spectra of similar differential operators considered below follows from Theorem 1 of paper [5] and from theorem about product of closed semi-Fredholm operators (see [12], Theorem IV.2.7).

Theorem 3.2. Let $X, Y$ and $Z$ be Banach spaces and a closed linear operator $T: X \rightarrow Y$ belongs to a class $\Phi^{+}$, i.e. $\overline{R(T)}=R(T)$ and $n u l(T)<\infty$. Let us assume, that $B: Z \rightarrow X$ is a linear operator. Then the following statements hold:

1. If operator $B$ is closed then $T B$ is closed operator.

2. If $B$ is a normally solvable, i.e. $\overline{R(T)}=R(T)$, then $T B$ is also normally solvable, i.e. $\overline{R(T B)}=R(T B)$.

3. If the domain of operator $T$ is dense in $X$ and operators $T$ and $B$ are Fredholm, then the operator $T B$ is Fredholm and

$$
\operatorname{ind}(T B)=\operatorname{ind}(T)+\operatorname{ind}(B)
$$

4. If the domains of operators $T$ and $B$ are dense in $X$ and $Z$, respectively, and the operator $B$ is closed and belongs to class $\Phi^{-}$, i.e. $\operatorname{def}(B)<\infty$, then the domain of product of operators $T B$ is dense $Z$. 
In order to study domains of differential operators similar to Fuchsian maximal and minimal differential operators, considered as perturbed Euler differential operators, we will use the following auxiliary statements (see. e.g., [15], statement 38.4, pages 214-215, and Statement 9.3, page 51, respectively).

Let function $f$ be integrable on a segment $[a, b]$ and let function $\varphi$ be absolutely continuous and monotone on a segment $[\alpha, \beta]$, such that $a \leq \varphi(t) \leq b$, for $\alpha \leq t \leq \beta$. Then function $f(\varphi(t)) \varphi^{\prime}(t)$ is integrable and the following equality holds:

$$
\int_{\varphi(\alpha)}^{\varphi(\beta)} f(x) d x=\int_{\alpha}^{\beta} f(\varphi(t)) \varphi^{\prime}(t) d t .
$$

Let function $f$ be integrable on a segment $[a, b]$ and let function $\varphi$ be defined and absolutely continuous on a segment $[\alpha, \beta]$ with values belonging to the interval $[a, b]$. Then the following two conditions are equivalent:

(i) the function $\varphi$ is monotone,

(ii) if the function $f$ satisfies Lipschitz condition, then $f(\varphi(t))$ is absolutely continuous on $[\alpha, \beta]$.

\section{ESSENTIAL SPECTRA OF FUSHSIAN DIFFERENTIAL OPERATORS}

In the following basic theorem using special assumptions on smallness in average on infinity for coefficients $b_{k}$ we prove the equalities for essential spectra of Fuchsian minimal, maximal and intermediate differential operators, generated by operation $\omega$, and for corresponding differential operators, generated by Euler operation $\varepsilon$, on Lebesgue spaces $L^{p}(a, \infty), 0<a<\infty, 1 \leq p<\infty$.

Theorem 4.1. Let coefficients $b_{k}$ of the formal ordinary differential operation $\omega$, defined by formula (2.1), satisfy conditions $b_{k} \in L_{l o c}^{p}(a, \infty), 0<a<\infty$, where $1 \leq p<\infty$, and let

$$
\lim _{s \rightarrow \infty} \int_{e^{s}}^{e^{s+1}} \frac{1}{t}\left|b_{k}(t)\right|^{p} d t=0, \quad k=\overline{0, n}
$$

Then for maximal $T(\omega, p,[a, \infty))$, minimal $T_{0}(\omega, p,[a, \infty))$ and closed $S(\omega, p,[a, \infty)), T_{0} \subseteq S \subseteq T$, Fuchsian differential operators, generated by formal operation

$$
\omega=\varepsilon+\sum_{k=0}^{n} b_{k}(t) t^{k} D^{k}
$$


Some results on essential spectra of diff. operators in Banach spaces 209

on the Lebesgue spaces $L^{p}(a, \infty), 0<a<\infty, 1 \leq p<\infty$, and for corresponding differential operators, defined by the Euler operation

$$
\varepsilon=\sum_{k=0}^{n} a_{k} t^{k} D^{k},
$$

the following equalities hold for the essential spectra:

$$
\begin{aligned}
& \sigma_{e k}[S(\omega, p,[a, \infty))]=\sigma_{e k}[S(\varepsilon, p,[a, \infty))], k=1,2,2^{ \pm}, 3, \\
& \sigma_{e k}\left[T_{0}(\omega, p,[a, \infty))\right]=\sigma_{e k}\left[T_{0}(\varepsilon, p,[a, \infty))\right], k=4,5, \\
& \sigma_{e k}[T(\omega, p,[a, \infty))]=\sigma_{e k}[T(\varepsilon, p,[a, \infty))], k=4,5 .
\end{aligned}
$$

Proof. Let us take the advantage of mapping $\Theta: L^{p}(a, \infty) \rightarrow L^{p}\left(a^{\star}, \infty\right)$, where $0<a<\infty, a^{\star}:=\ln a$, which is defined for all $f \in L^{p}(a, \infty), 1 \leq p<\infty$ by the following formula

$$
(\Theta f)(s):=e^{s / p} f\left(e^{s}\right)=g(s), \quad a^{\star} \leq s<\infty .
$$

The inverse mapping $\Theta^{-1}: L^{p}\left(a^{\star}, \infty\right) \rightarrow L^{p}(a, \infty)$ is given by the formula

$$
\left(\Theta^{-1} g\right)(t):=t^{-1 / p} g(\ln t)=f(t), \quad a \leq t<\infty
$$

for all functions $g \in L^{p}\left(a^{\star}, \infty\right), 1 \leq p<\infty$.

It follows from auxiliary statements that spaces $L^{p}(a, \infty)$ and $L^{p}\left(a^{\star}, \infty\right)$ are equivalent with respect to the mapping $\Theta$. Since function $e^{s}$ on $\left[a^{\star}, \infty\right)$ and function $\ln t$ on $[a, \infty)$ are monotonically growing and indefinitely differentiable, then we obtain from formulas (4.3), (4.4) that

$$
\Theta\left(A C^{n}[a, \infty) \cap L^{p}(a, \infty)\right)=A C^{n}\left[a^{\star}, \infty\right) \cap L^{p}\left(a^{\star}, \infty\right) .
$$

Here $A C^{n}(I)$ is a space of complex-valued functions $f$ defined on set $I \subseteq \mathbf{R}$, for which the derivative $f^{(n-1)}=D^{n-1} f$ exists and it is absolutely continuous in each compact sub-interval from $I$. If the function $f$ is from space $A C^{n}[a, \infty)$, then $g=\Theta f \in A C^{n}\left[a^{\star}, \infty\right)$ and hence, by equality (4.4), we obtain:

$$
\begin{gathered}
\frac{d f}{d t}=t^{-(1 / p)-1} g^{\prime}(\ln t)-(1 / p) t^{-(1 / p)-1} g(\ln t) \\
=t^{-(1 / p)-1}\left[\left(\frac{d}{d t}-\frac{1}{p}\right) g(s)\right]_{s=\ln t}, \\
\frac{d^{2} f}{d t^{2}}=t^{-(1 / p)-2} \frac{d}{d s}\left[\left(\frac{d}{d s}-\frac{1}{p}\right) g(s)\right]_{s=\ln t}-\left(\frac{1}{p}+1\right) t^{-(1 / p)-2} \\
\times\left[\left(\frac{d}{d s}-\frac{1}{p}\right) g(s)\right]_{s=\ln t}=t^{-(1 / p)-2}\left[\left(\frac{d}{d s}-\frac{1}{p}-1\right)\left(\frac{d}{d s}-\frac{1}{p}\right) g(s)\right]_{s=\ln t} .
\end{gathered}
$$


Continuing this process by an induction for $1 \leq k \leq n$ we have:

$$
\begin{aligned}
\frac{d^{k} f}{d t^{k}}=t^{-(1 / p)-k}\left[\left(\frac{d}{d s}-\left(\frac{1}{p}+k-1\right)\right)\right. & \left(\frac{d}{d s}-\left(\frac{1}{p}+k-2\right)\right) \\
& \left.\times \cdots\left(\frac{d}{d s}-\frac{1}{p}\right) g(s)\right]_{s=\ln t} .
\end{aligned}
$$

Therefore for functions $f \in D[T(\omega, p,[a, \infty))]$ the following equalities hold:

$$
\begin{gathered}
(\varepsilon f)(t)=\left.t^{-1 / p}\left[a_{0}+\sum_{k=1}^{n} a_{k} \prod_{j=0}^{k-1}\left(\frac{d}{d s}-\left(\frac{1}{p}+j\right)\right)\right] g(s)\right|_{s=\ln t}, \\
(\omega f)(t)=t^{-1 / p}\left[a_{0}+b_{0}\left(e^{s}\right)+\sum_{k=1}^{n}\left(a_{k}+b_{k}\left(e^{s}\right)\right) \prod_{j=0}^{k-1}\left(\frac{d}{d s}\right.\right. \\
\left.\left.-\left(\frac{1}{p}+j\right)\right)\right]\left.g(s)\right|_{s=\ln t} .
\end{gathered}
$$

Let us consider ordinary differential operations $\alpha$ and $\beta$, defined as

$$
\begin{aligned}
& \alpha:=a_{0}+\sum_{k=1}^{n} a_{k} \prod_{j=0}^{k-1}\left[\frac{d}{d s}-\left(\frac{1}{p}+j\right)\right] \\
& \beta:=\alpha+b_{0}\left(e^{s}\right)+\sum_{k=1}^{n} b_{k}\left(e^{s}\right) \prod_{j=0}^{k-1}\left[\frac{d}{d s}-\left(\frac{1}{p}+j\right)\right] .
\end{aligned}
$$

Let $T\left(\alpha, p,\left[a^{\star}, \infty\right)\right)$ and $T\left(\beta, p,\left[a^{\star}, \infty\right)\right)$ be maximal differential operators, corresponding to operations $\alpha, \beta$, defined by formulas (4.8), with domains, contained in space $L^{p}\left(a^{\star}, \infty\right)$. It follows from Eqs. (4.6), (4.7) and from formula (4.3) that

$$
\Theta(\varepsilon f)=\alpha g=\alpha(\Theta f), \quad \Theta(\omega f)=\beta g=\beta(\Theta f)
$$

Therefore in virtue of equality (4.5) and formulas (4.9) for appropriate maximal differential operators we obtain

$$
\begin{aligned}
& T(\varepsilon, p,[a, \infty))=\Theta^{-1} T\left(\alpha, p,\left[a^{\star}, \infty\right)\right) \Theta, \\
& T(\omega, p,[a, \infty))=\Theta^{-1} T\left(\beta, p,\left[a^{\star}, \infty\right)\right) \Theta,
\end{aligned}
$$

which are equivalent to equalities

$$
\begin{aligned}
& T(\varepsilon, p,[a, \infty))-\lambda I=\Theta^{-1}\left(T\left(\alpha, p,\left[a^{\star}, \infty\right)\right)-\lambda I\right) \Theta \\
& T(\omega, p,[a, \infty))-\lambda I=\Theta^{-1}\left(T\left(\beta, p,\left[a^{\star}, \infty\right)\right)-\lambda I\right) \Theta .
\end{aligned}
$$


These equalities hold for any $\lambda \in \mathbf{C}$.

The mapping $\Theta$ is a linear isometry. From equalities (4.10), in virtue of Theorem 2 and Theorem 1 [5], we obtain the coincidence of essential spectra of Euler maximal differential operators and Fuchsian maximal differential operators with special differential operators with constant coefficients and perturbed differential operators with almost constant coefficients. Thus, taking into account the equality

$$
\int_{x}^{x+1}\left|b_{k}\left(e^{s}\right)\right|^{p} d s=\int_{e^{x}}^{e^{x+1}} \frac{1}{t}\left|b_{k}(t)\right|^{p} d t, 0 \leq k \leq n,
$$

in virtue of conditions (4.1) on functions $b_{k}$, included into coefficients of Fuchsian differential operator from Th. 3.1 the theorem follows for maximal differential operators. In the same way equalities for essential spectra of minimal differential operators are proved. From Theorem 1 [5], where results of coincidence of essential spectra of differential operators with smooth coefficients are formulated, spectral correlation for the appropriate intermediate operators follow.

For the proof of the analog of Thm. 4.1 for Fuchsian minimal and maximal differential operators on Lebesgue space $L^{\infty}(a, \infty), 0<a<\infty$, the equality for adjoint of the product of the densely defined operators and for the product of their adjoint operators is necessary. In general the inequality $(S T)^{\prime} \neq T^{\prime} S^{\prime}$ holds for unbounded densely defined operators on Banach spaces (see the corresponding counter-example in [11], p. 304-305). Schechter [19] proved the equality for adjoint operators $(S T)^{\prime}=T^{\prime} S^{\prime}$ provided that the operator $T$ is semi-Fredholm operator from $\Phi^{-}$. His result is formulated in the following lemma.

Lemma 4.1. Let $X, Y$ and $Z$ be Banach spaces. We assume that a closed defined linear operator $T: X \rightarrow Y$ is semi-Fredholm from $\Phi^{-}$, that is $\overline{R(T)}=$ $R(T)$ and

$$
\operatorname{def}(T)=\operatorname{dim}[Y / R(T)]<\infty .
$$

Then if $S: Y \rightarrow Z$ is densely defined linear operator, then $D(S T)$ is dense in $X$ and the equality $(S T)^{\prime}=T^{\prime} S^{\prime}$ holds for adjoint product of operators.

In Theorem 1 of paper [16] it is proved that belonging of the operator $T$ to $\Phi^{-}$is a necessary condition.

Theorem 4.2. Let coefficients $b_{k}$ in (2.1) and their derivatives $b_{k}^{(i)}, i=\overline{1, k}$ satisfy the integral condition (4.1) for $p=1$. Then for essential spectra of Fuchsian minimal $T_{0}(\omega, \infty,[a, \infty))$, maximal $T(\omega, \infty,[a, \infty))$ and closed intermediate $S(\omega, \infty,[a, \infty))$ differential operators, generated by perturbed ope- 
ration $\omega$ on Lebesgue space $L^{\infty}(a, \infty), 0<a<\infty$, the equalities (4.2) hold, where $p=\infty$.

Proof. Let us consider the duality correlation for minimal and maximal operators, generated by differential operation $\omega$, defined by formula (2.1) on Banach space $L^{\infty}(a, \infty)$, and Banach adjoint operators to maximal and minimal operators generated by formally adjoint differential operation $\omega^{*}$, defined by formula $(2.2)$ on Banach space $L^{1}(a, \infty)$. Thus we take the equalities such as (see $[5 ; 12])$

$$
\begin{aligned}
& T_{0}(\omega, \infty,[a, \infty))=T^{\prime}\left(\omega^{*}, 1,[a, \infty)\right), \\
& T(\omega, \infty,[a, \infty))=T_{0}^{\prime}\left(\omega^{*}, 1,[a, \infty)\right) .
\end{aligned}
$$

Analogous duality equalities are obtained for minimal and maximal differential operators, generated by Euler operation $\varepsilon$ and formally adjoint operation

$$
\varepsilon^{*} g:=\sum_{k=0}^{n}(-1)^{k} D^{k}\left(a_{k} t^{k} g\right)
$$

in the corresponding spaces $L^{\infty}(a, \infty)$ and $L^{1}(a, \infty)$.

From the duality formulas (4.11) we obtain, that differential operators $T\left(\omega^{*}, 1,[a, \infty)\right)$ and $T_{0}\left(\omega^{*}, 1,[a, \infty)\right)$ can be considered as preconjugate corresponding to differential operators $T_{0}(\omega, \infty,[a, \infty))$ and $T(\omega, \infty,[a, \infty))$. Let us note that for the adjoint to the bounded inverse $\Theta^{-1}$ of bounded operator $\Theta$ the equality $\left(\Theta^{-1}\right)^{\prime}=\left(\Theta^{\prime}\right)^{-1}$ holds. Similarly to (4.10), we obtain the equalities formulated for the formally adjoint differential operations $\varepsilon^{*}, \omega^{*}$ and $p=1$

$$
\begin{aligned}
& T\left(\varepsilon^{*}, 1,[a, \infty)\right)-\lambda I=\Theta^{-1}\left(T\left(\alpha, 1,\left[a^{\star}, \infty\right)\right)-\lambda I\right) \Theta \\
& T\left(\omega^{*}, 1,[a, \infty)\right)-\lambda I=\Theta^{-1}\left(T\left(\beta, 1,\left[a^{\star}, \infty\right)\right)-\lambda I\right) \Theta
\end{aligned}
$$

where $\alpha$ and $\beta$ are differential operations with constant coefficients, defined by formulas (4.8). Applying for them the formulas of duality (4.11) for operations $\omega, \varepsilon$, using Lemma 4.1 and Th. 3.2, we obtain, with help of Th. 4.1 (see also [7; $9]$ ), the required equalities for essential spectra of Fuchsian minimal differential operator $T_{0}(\omega, \infty,[a, \infty))$. In the same way, using analog of equalities (4.12) for minimal operators the statement of the theorem for maximal operators $T(\omega, \infty,[a, \infty))$ and in virtue of Theorem 1 [5] for the intermediate operator $S(\omega, \infty,[a, \infty))$ can be proved.

\section{EULER DIFFERENTIAL OPERATORS}

Let us formulate the result about essential spectra of Euler differential operators [6]. 
Lemma 5.1. The essential spectra and spectra of the Euler minimal differential operators $T_{0}(\varepsilon, p,[a, \infty))$, maximal operator $T(\varepsilon, p,[a, \infty))$ and intermediate operator $S(\varepsilon, p,[a, \infty))$, generated on $L^{p}(a, \infty), 0<a<\infty, 1 \leq p \leq \infty$, by the operation $\varepsilon=\sum_{k=0}^{n} a_{k} t^{k} D^{k}$, are $p$-dependent and calculated as

$$
\begin{aligned}
& \sigma_{e k}[S(\varepsilon, p,[a, \infty))]=\sigma_{e 2}^{ \pm}[S(\varepsilon, p,[a, \infty))]=\{Q(\lambda): \operatorname{Re} \lambda=0\}, k=1,2,3, \\
& \sigma_{e k}\left[T_{0}(\varepsilon, p,[a, \infty))\right]=\sigma\left[T_{0}(\varepsilon, p,[a, \infty))\right]=\{Q(\lambda): \operatorname{Re} \lambda \geq 0\}, k=4,5,(5.1) \\
& \sigma_{e k}[T(\varepsilon, p,[a, \infty))]=\sigma[T(\varepsilon, p,[a, \infty))]=\{Q(\lambda): \operatorname{Re} \lambda \leq 0\}, k=4,5,
\end{aligned}
$$

where $Q$ is a polygon, appropriate to differential operation $\alpha$ (4.8), defined by the equality:

$$
Q(t):=a_{0}+\sum_{k=1}^{n} a_{k} \prod_{j=0}^{k-1}\left[t-\left(\frac{1}{p}+j\right)\right] .
$$

One can use the proved Th. 4.1 and Th.4.2 for finding the exact formulas of various types of Fuchsian differential operators on Lebesgue spaces $L^{p}(a, \infty), 1 \leq p \leq \infty$.

Theorem 5.1. Under conditions on coefficients $b_{k}$ of differential operation $\omega$ from Th. 4.1 for $1 \leq p<\infty$ and Th. 4.2 for $p=\infty$, for essential spectra and spectra of Fuchsian minimal, maximal and intermediate differential operators, defined on $L^{p}(a, \infty), 0<a<\infty$, for all $1 \leq p \leq \infty$, formulas as (5.1) are valid, where operation $\omega$ should be written instead of $\varepsilon$.

The proof of the statement follows from the equality for essential spectra of the corresponding Fuchsian differential operators, obtained in Th. 4.1 and Th. 4.2 and from Lemma 5.1.

As application of Th. 5.1 let us describe all essential spectra of Riemman differential operators, that for the first time was considered in [18] and then in $[2 ; 12]$. We consider minimal $T_{0}(r, p,[a, \infty))$, maximal $T(r, p,[a, \infty))$ and intermediate $T(r, p,[a, \infty))$ differential operators, generated on Lebesgue spaces $L^{p}(a, \infty), 1 \leq p \leq \infty$, by Riemann differential operation $r$ such as

$$
(r f)(t):=t(t+1) f^{(2)}(t)+(a t+b) f^{\prime}(t)+\frac{c t^{2}+d t+e}{t(t+1)} f(t),
$$

where $a, b, c, d, e$ are arbitrary complex numbers.

Theorem 5.2. For essential spectra $\sigma_{e k}, k=\overline{1,5}$ and $\sigma_{e 2}^{ \pm}$of Riemann differential operators, generated on Lebesgue spaces $L^{p}(a, \infty), 1 \leq p \leq \infty$, by the formal operation $r$ (5.3), the formulas such as (5.1) hold, where $r$ should be replaced by $\varepsilon$ and

$$
Q(t)=(t-1 / p)(t-(1 / p)-1)+a(t-1 / p)+c .
$$


Proof. This statement follows from Th. 5.1. The differential operation (5.3) can be represented as

$$
(r f)(t)=t^{2}\left(1+b_{2}(t)\right) f^{(2)}(t)+t\left(a+b_{1}(t)\right) f^{\prime}(t)+\left(c+b_{0}(t)\right) f(t),
$$

with coefficients, defined by formulas

$$
b_{2}(t)=\frac{1}{t}, \quad b_{1}(t)=\frac{b}{t}, \quad b_{0}(t)=\frac{d-c}{t+1}+\frac{e}{t(t+1)} .
$$

Let us note, that mapping $(\Theta f)(s)=e^{s / p} f\left(e^{s}\right)$, defined in the proof of Th. 4.1, also establishes isometric isomorphism of $L^{p}(0, a), 0<a<\infty$ and $L^{p}\left(-\infty, a^{\star}\right)$, where $a^{\star}=\ln a$. Therefore Fuchsian maximal differential operator $T(\omega, p,(0, a])$, defined by formula $(2.1)$ on space $L^{p}(0, a)$, is similar through $\Theta$ to maximal differential operator $T\left(\beta, p,\left(-\infty, a^{\star}\right]\right)$, defined by the formula (4.8) on space $L^{p}\left(-\infty, a^{\star}\right)$. The similar statement is valid for the corresponding minimal operators.

Since

$$
D \nu f(t)=(-D f)(-t)=\nu(-D f)(t),
$$

that is $\nu^{-1} D \nu=-D$, the operator $D=d / d t$ on space $L^{p}\left(-\infty, a^{\star}\right)$ is similar isometric mapping $\nu f(t):=f(-t)$ to operator $(-D)$ in space $L^{p}\left(-a^{\star}, \infty\right)$. Therefore maximal differential operator $T\left(D, p,\left(-\infty, a^{\star}\right]\right)$ is similar to maximal differential operator $T\left(-D, p,\left[-a^{\star}, \infty\right)\right)$. The same is valid for the corresponding minimal differential operators.

Theorem 5.3. Let $T_{0}(\omega, p,(0, a])$ and $T(\omega, p,(0, a])$ be Fuchsian minimal and maximal differential operators, generated on Lebesgue spaces $L^{p}(0, a), 0<a<$ $\infty, 1 \leq p \leq \infty$, by operation $\omega(2.1)$, and let coefficients $b_{k}$ satisfy conditions of Th. 4.1 for $1 \leq p<\infty$ and of Th. 4.2 for $p=\infty$. Then essential spectra of minimal, maximal and closed intermediate $S(\omega, p,(0, a]), T_{0} \subseteq S \subseteq T$, differential operators are $p$-dependent and defined by:

$$
\begin{aligned}
& \sigma_{e k}[S(\omega, p,(0, a])]=\sigma_{e 2}^{ \pm}[S(\omega, p,(0, a])]=\{Q(\lambda): \operatorname{Re} \lambda=0\}, k=1,2,3 \\
& \sigma_{e k}\left[T_{0}(\omega, p,(0, a])\right]=\sigma\left[T_{0}(\omega, p,(0, a])\right]=\{Q(\lambda): \operatorname{Re} \lambda \leq 0\}, k=4,5, \\
& \sigma_{e k}[T(\omega, p,(0, a])]=\sigma[T(\omega, p,(0, a])]=\{Q(\lambda): \operatorname{Re} \lambda \geq 0\}, k=4,5,
\end{aligned}
$$

where $Q$ is a polygon, defined by (5.2).

The proof follows from the analog of the Th. 5.1 and the Th. 3.2 and Th.4.1. The difference between formulas (5.4) and (5.1) is due to the fact, that although for the Fredholm essential spectra they coincide, since

$$
\sigma_{e 3}\left[T_{0}\left(D, p,\left(-\infty, a^{\star}\right]\right)\right]=\sigma_{e 3}\left[T\left(D, p,\left(-\infty, a^{\star}\right]\right)\right]=\{\lambda \in \mathbf{C}: \operatorname{Re} \lambda=0,\}
$$


the spectra of minimal and maximal differential operators are equal to

$$
\begin{aligned}
& \sigma\left[T_{0}\left(D, p,\left(-\infty, a^{\star}\right]\right)\right]=\{\lambda \in \mathbf{C}: \operatorname{Re} \lambda \leq 0\}, \\
& \sigma\left[T\left(D, p,\left(-\infty, a^{\star}\right]\right)\right]=\{\lambda \in \mathbf{C}: \operatorname{Re} \lambda \geq 0\},
\end{aligned}
$$

respectively.

\section{CONCLUSIONS}

Let us note in conclusion that in the monograph [17] and papers $[1 ; 10 ; 14]$ the structural and spectral properties of Euler self-adjoint differential operator and some their perturbations on Hilbert space were studied. In the work [4] the Fredholm properties of Fuchsian differential operators such as

$$
(A y)(x)=\left(x^{l} D^{l}+\sum_{0 \leq i<l} a_{i}(x) x^{i} D^{i}\right) y(x)
$$

on the finite interval with singularity in the point $x=0$ in special classes of Sobolev spaces were investigated.

\section{REFERENCES}

[1] E. Balslev. The singular spectrum of elliptic differential operators in $L^{p}\left(R_{n}\right)$. Math. Scand., 19, 193 - 210, 1966.

[2] E. Balslev and Gamelin T.W. The essential spectrum of a class of ordinary differential operators. Pacific J. Math., 14, 755 - 776, 1964.

[3] D.E. Edmunds and Evans W.D. Spectral theory and differential operators. Oxford, 1987.

[4] J. Elschner. Singular ordinary differential operators and pseudodifferential equations. Berlin, 1985.

[5] V.A. Erovenko. $L^{p}$-essential spectra of some classes of nonself-adjoint ordinary differential operators I. Operators with sufficiently smooth coefficients. Different. Equations. February, 1030 - 1039, 1997.

[6] V.A. Erovenko. $L^{p}$-essential spectra of some classes of nonself-adjoint ordinary differential operators II. Operators with constant coefficients and Euler operators. Different. Equations. March, 1164 - 1172, 1997.

[7] V.A. Erovenko. $L^{p}$-essential spectral theory of ordinary differential operators with almost constant coefficients. Matemat. Vesnik, 49, 177 - 185, 1997.

[8] V.A. Erovenko. Application of the abstract perturbation theory to the investigation of the essential spectra of ordinary differential operators in $L^{p}$. Different. Equations. January, $871-878,1998$.

[9] V.A. Erovenko. Stability of $L^{p}$-essential spectra of ordinary differential operators with almost constant coefficients. Different. Equations. July, 57 - 63, 1999.

[10] W.D. Evans, Kwong M.K. and Zettl A. Lower bounds for the spectrum of ordinary differential operators. J. Differ. Equat., 48, 123 - 155, 1983.

[11] K.-H. Förster and Liebetrau E.-O. On semi-Fredholm operators and conjugate of a product of operators. Stud. Math., 59, $301-306,1977$. 
[12] S. Goldberg. Unbounded linear operators. Theory and application. N.Y., 1966.

[13] T. Kato. Perturbation theory for linear operators. Springer, Berlin, 1966.

[14] R.M. Kauffman. On the limit-n classification of ordinary differential operators with positive coefficients. Proc. London Math. Soc., 135, 496 - 526, 1977.

[15] E.J. McShane. Integration. Princeton, 1947.

[16] S. Miyajima. Some converses of a theorem of M. Schechter on the conjugate of a product of operators. Sci. Pap. Coll. Gen. Educ. Univ. Tokyo, 32, 79-86, 1982.

[17] E. Müller-Pfeiffer. Spectral theory of ordinary differential operators. N.Y, 1981.

[18] G.-C. Rota. On the spectra of singular boundary value problems. J. Math. Mech., 10.

[19] M. Schechter. The conjugate of a product of operator. J. Functional Anal., 6, 26 - 28, 1970.

[20] M. Schechter. Spectra of partial differential operators. Amsterdam, 1971.

\section{Keletas rezultatu apie esminị spektrą Banacho erdvėse}

\section{V.A. Erovenko}

Šiame straipsnyje tiriamos spektrinès ir pusiau Fredholminès maksimalių ir minimalių Fuchsiano diferencialinių operatorių savybès Lebego erdvių pusiau ašyse. Šios savybès gali būti taikomos ivvairiems paprastųju diferencialinių operatorių su polinominiais koeficientais, kurių eilè neviršija atitinkamos išvestinès eilès, esminio spektro ir spektro tyrime. 